\title{
Trenchless Pipe Replacement
}

Trygve (Tryg) Hoff, PE ACPA Northeast Region Engineer

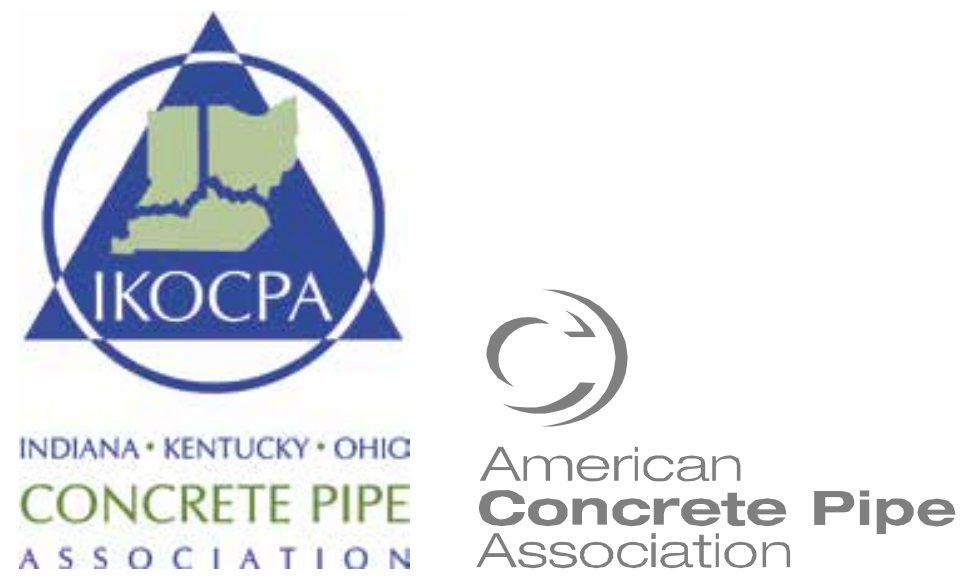




\section{Pipeline Functions}

What 2 functions must an underground pipe provide?

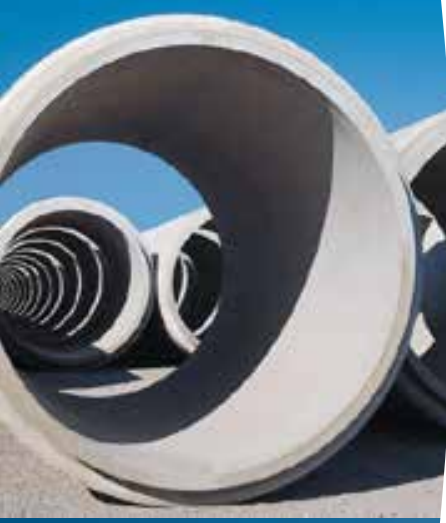

Conduit

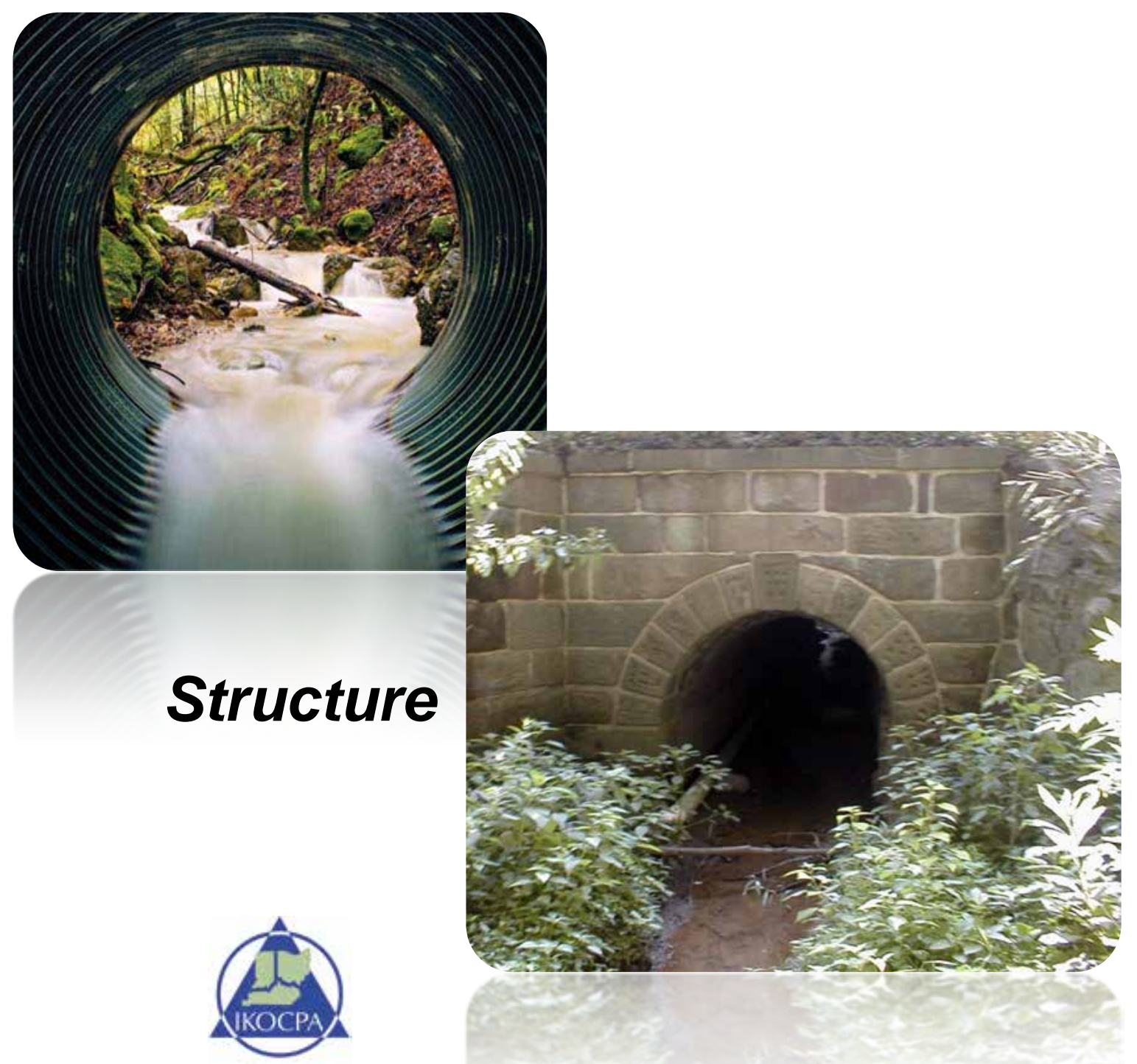




\section{Jacking Pipe - Replacement:}

\section{How does it work?}
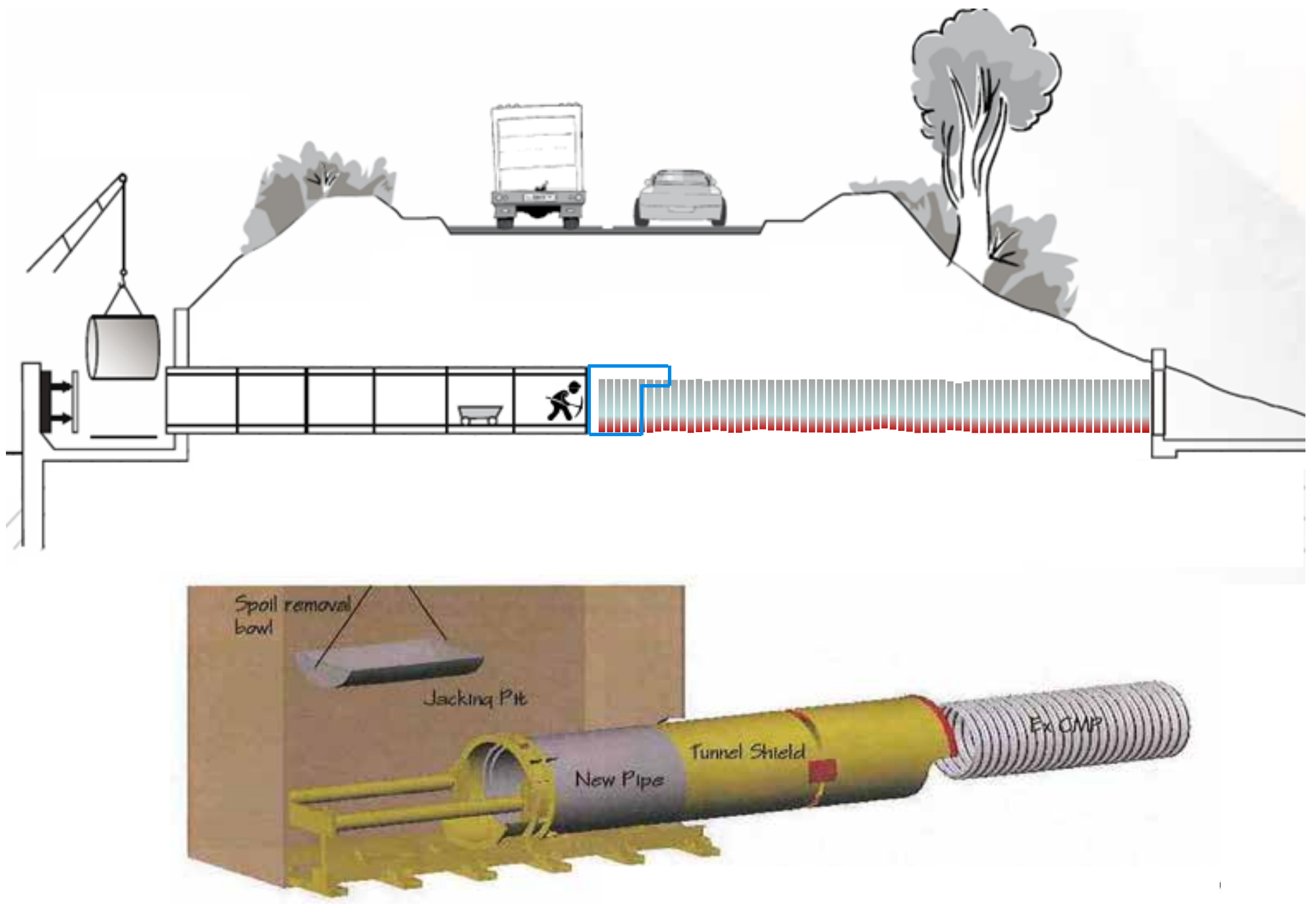


\section{Jacking Pipe - Replacement:}

\section{How does it work?}

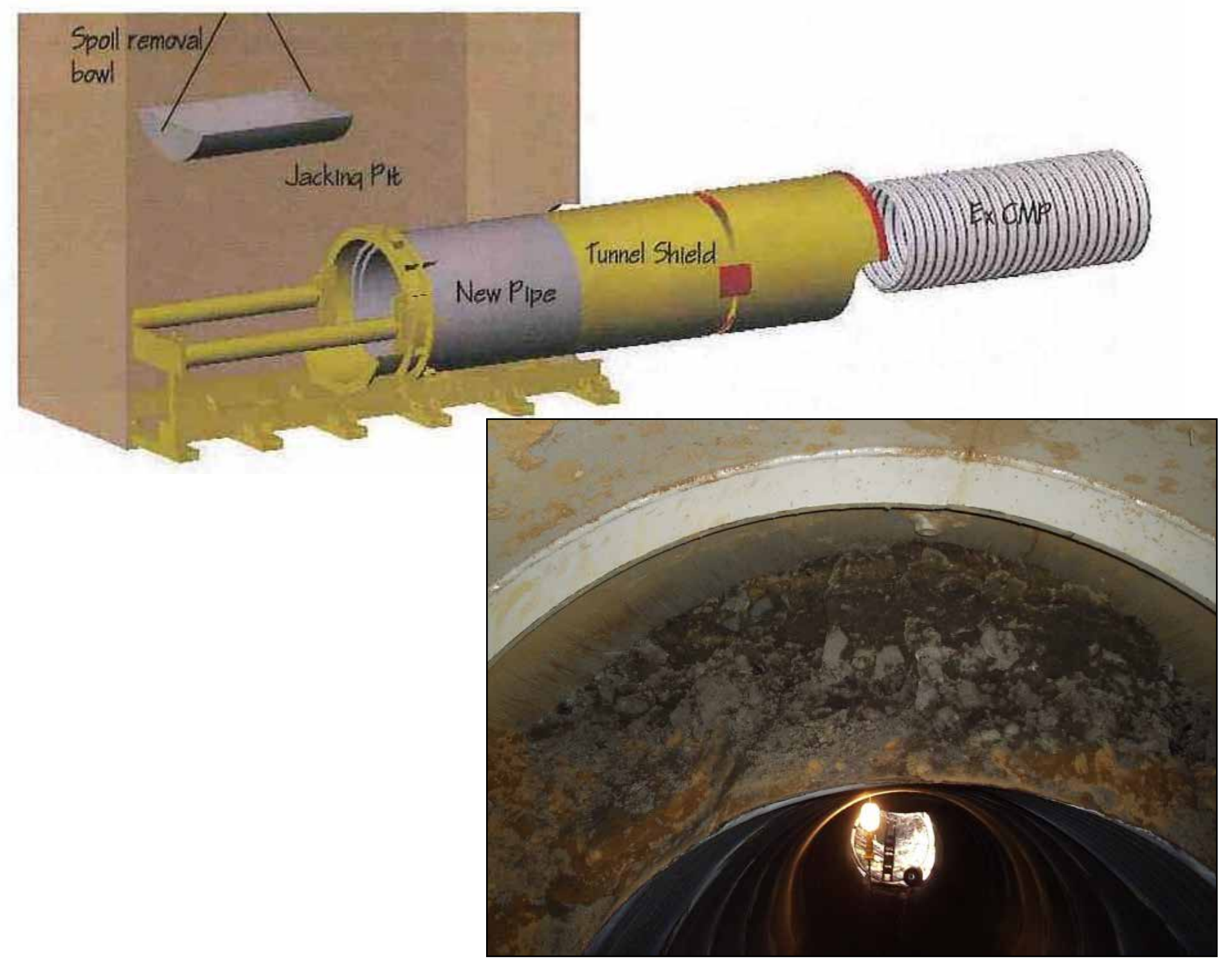




\section{Case Study: \\ Delaware DOT}

Delaware Route 4 Trenchless Pipe Replacement

- First use of tunneling replacement method with RCP jacking pipe in DelDOT's history

54" RCP culvert replaced 48" failing CMP

- Rinker Materials RCP with steel bands and grouting ports to withstand $400,000 \mathrm{lb}$ jacking force. 


\section{A) Case Study: DelDOT \\ Delaware DOT}

Delaware Route 4 Trenchless Pipe Replacement

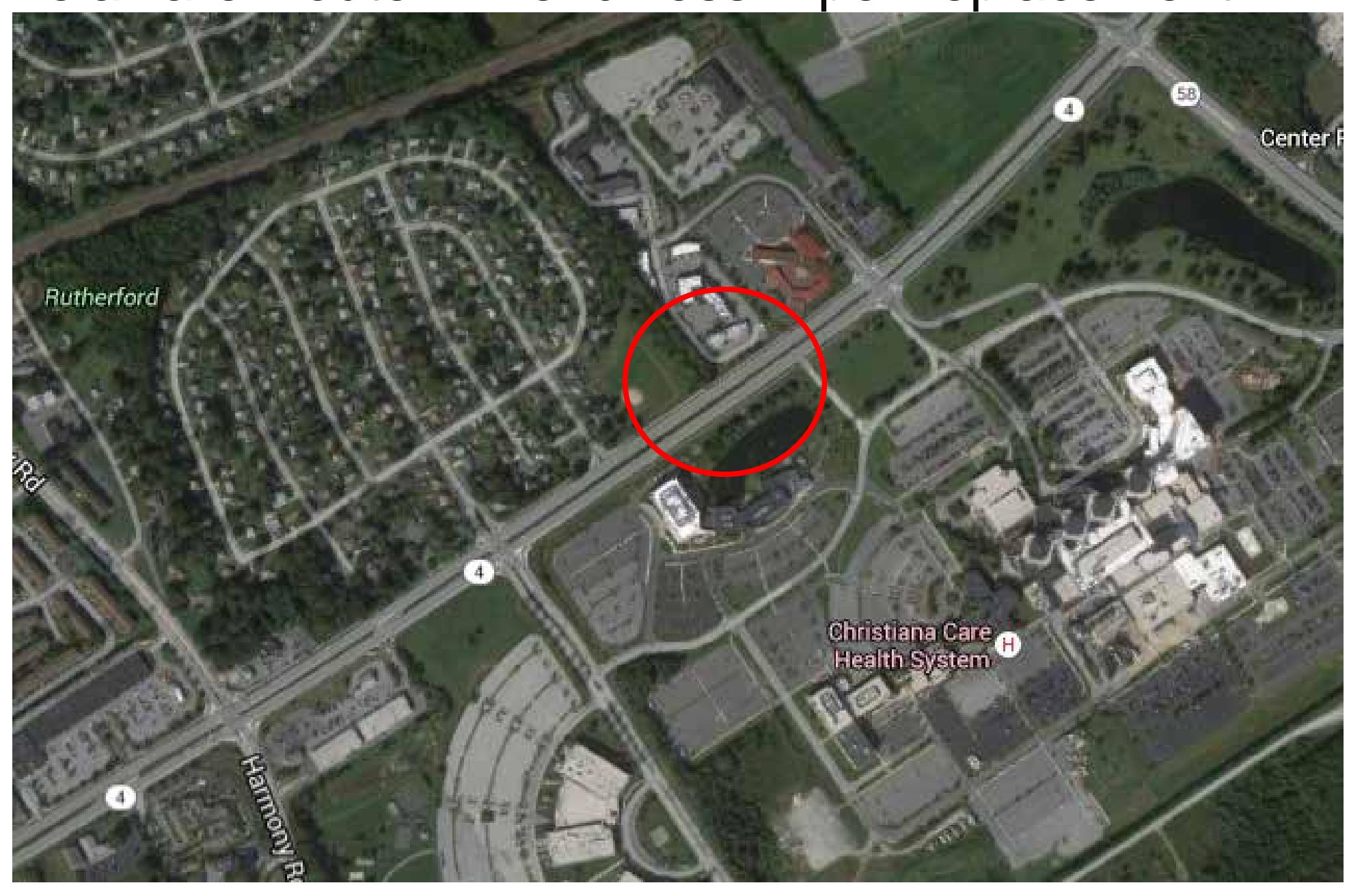




\section{SR 4: Proposed Plan}

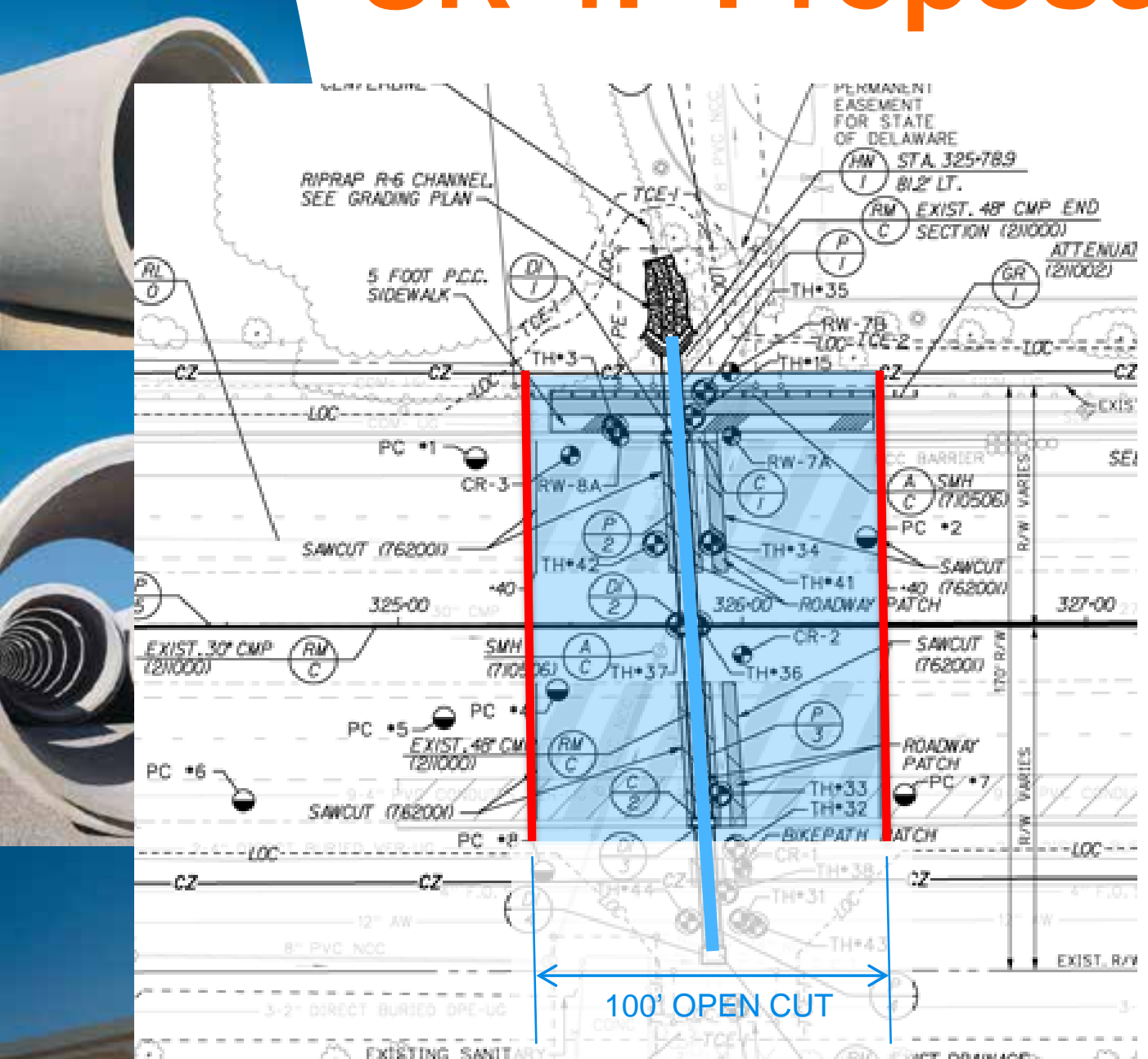

INITIAL OPEN CUT PLAN

y $~ 200$ ' replacement of failed corrugated metal pipe

y 100' Wide open cut trench

y 75 day project duration

- MOT Detours

- Stormwater rerouting

- Relocation of utilities
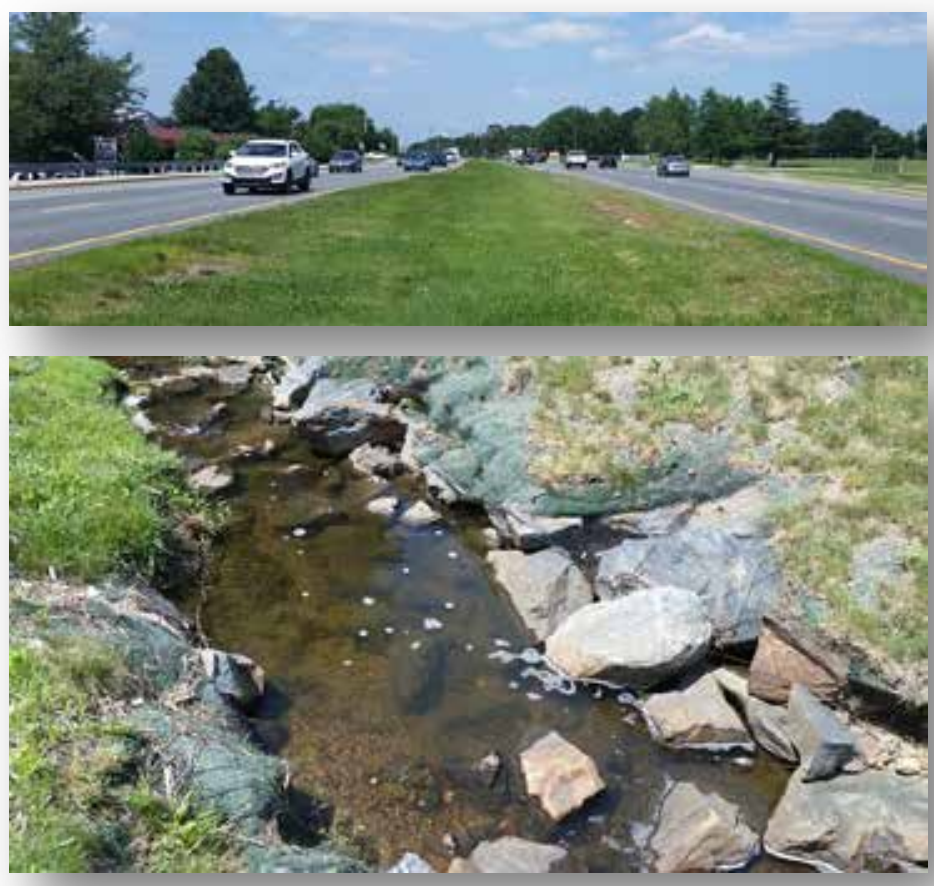


\section{Value Engineering Proposal}

Contractor proposed Jack \& Tunnel in lieu of Open-Cut

Advantages:

ÿ Cost Savings - $\$ 126,330$

(split between DelDOT \& Contractor)

ÿ Reduced M.O.T. and No Lane Shifts

ÿ Increased Work Zone Safety

ÿ No Supporting of Existing Utilities Required

ÿ Same contract duration (75 days)
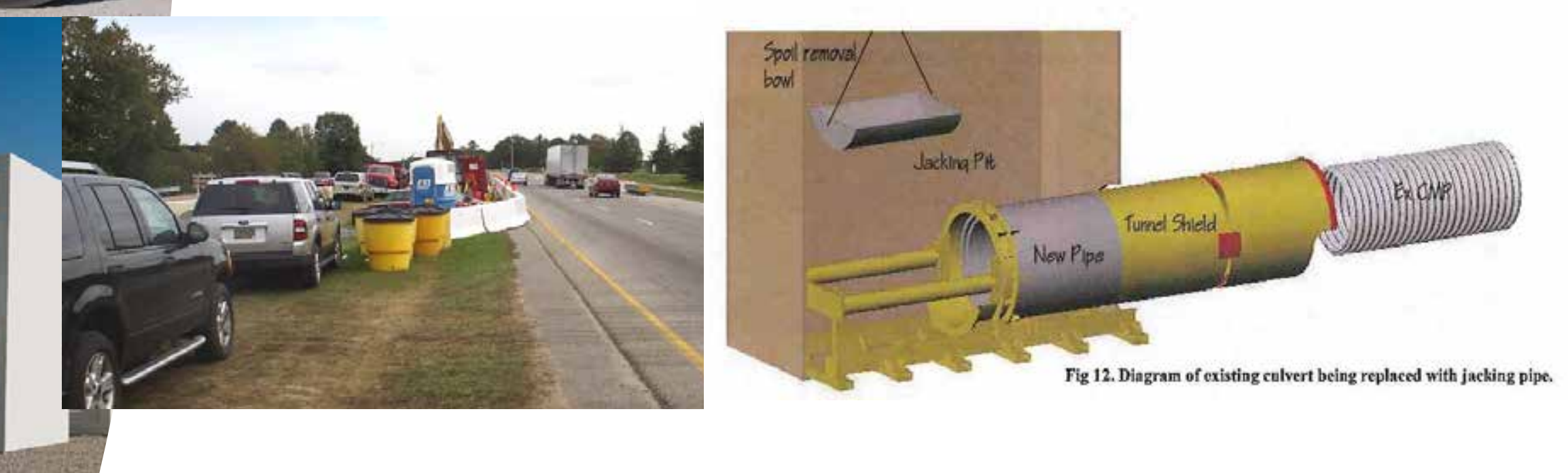


\section{Pipe Removal \& Jacking}
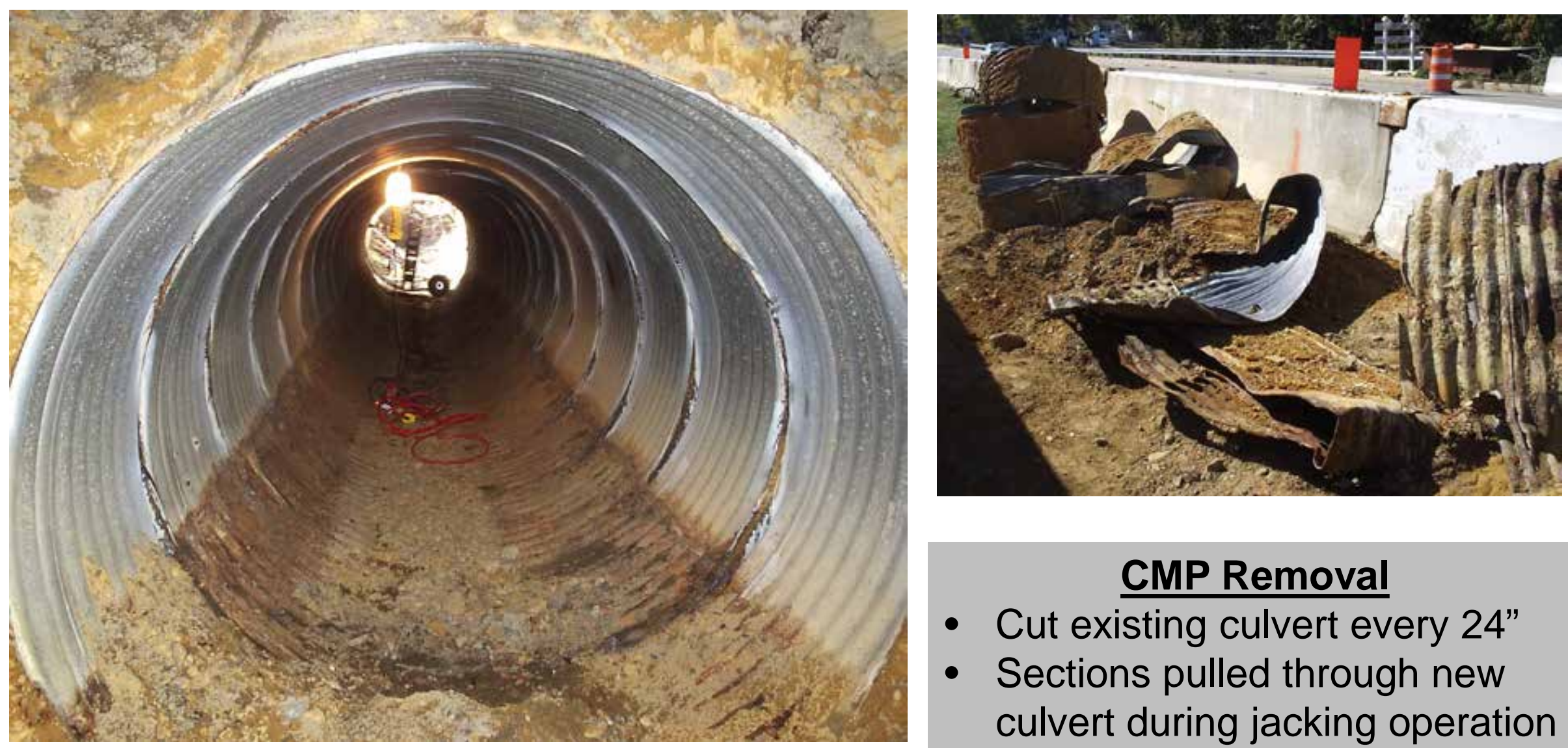

\section{CMP Removal}

- Cut existing culvert every 24"

- Sections pulled through new culvert during jacking operation

\section{Existing CMP}

- Deteriorated seams led to backfill infiltration

- Scour \& corrosion caused corroded invert

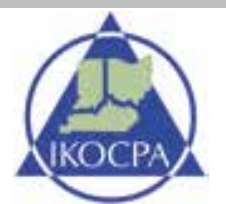




\section{Pipe Removal \& Jacking}
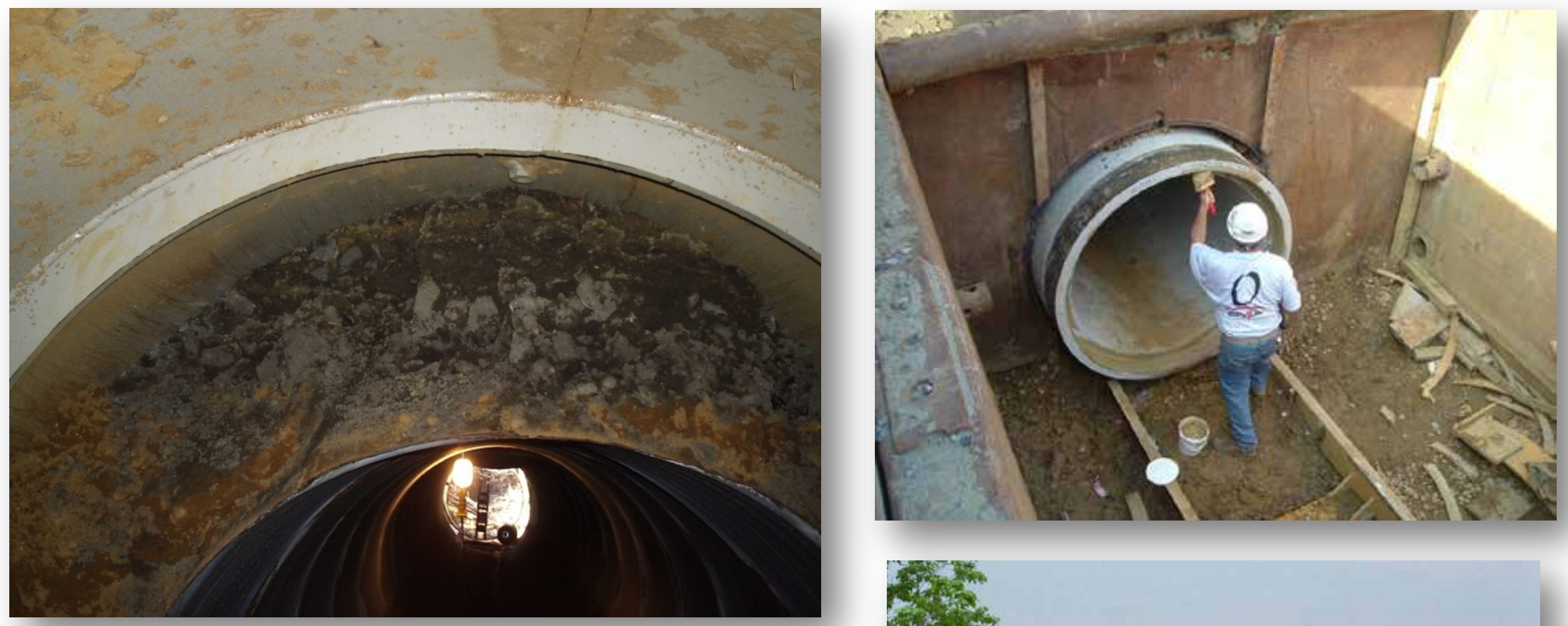

\section{Trenchless Installation}

- Minimize Impact to Drivers

- Increase Safety of Contractors

- Reduce Construction Time

- Shield sized larger than pipe OD

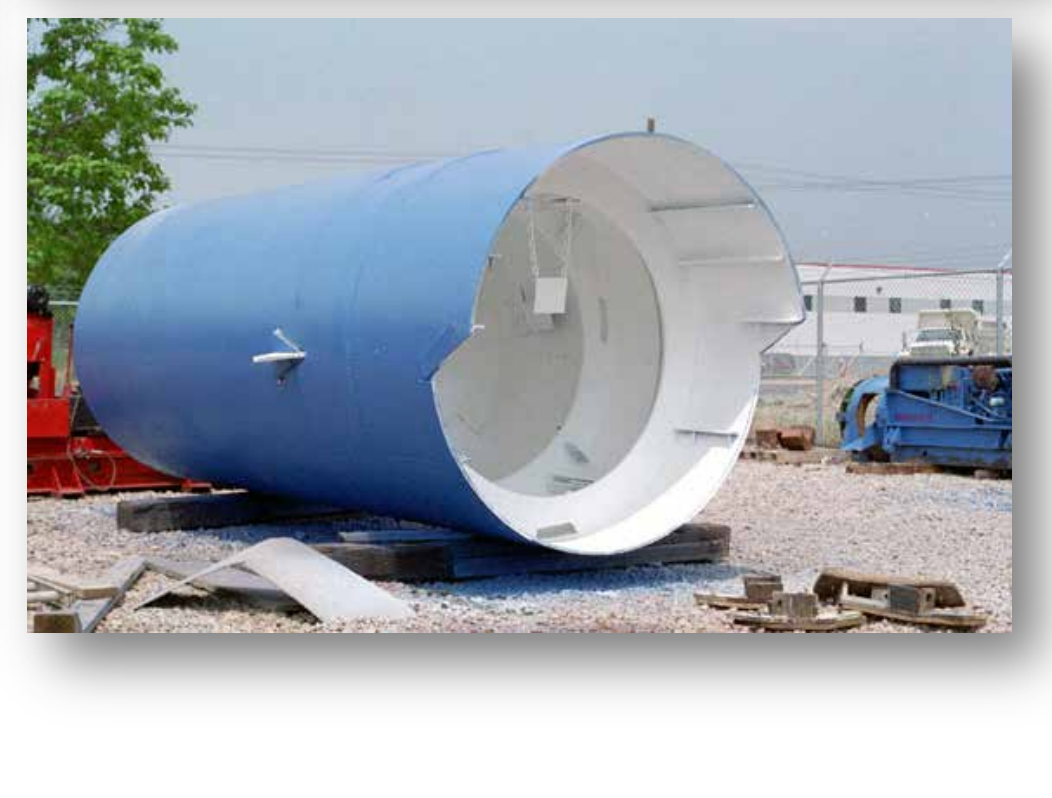




\section{Tunneling Operation}

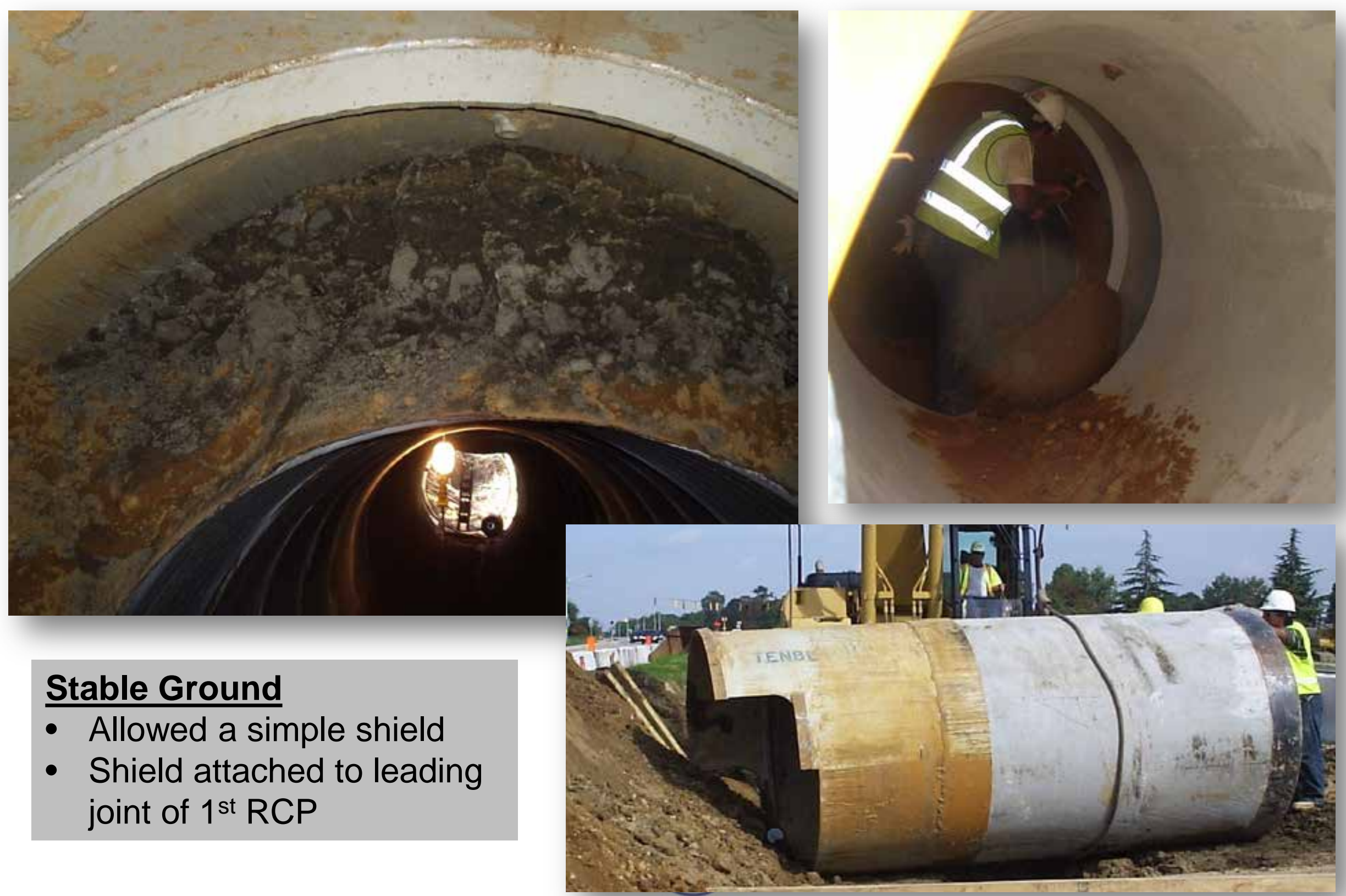




\section{Tunneling Operation}

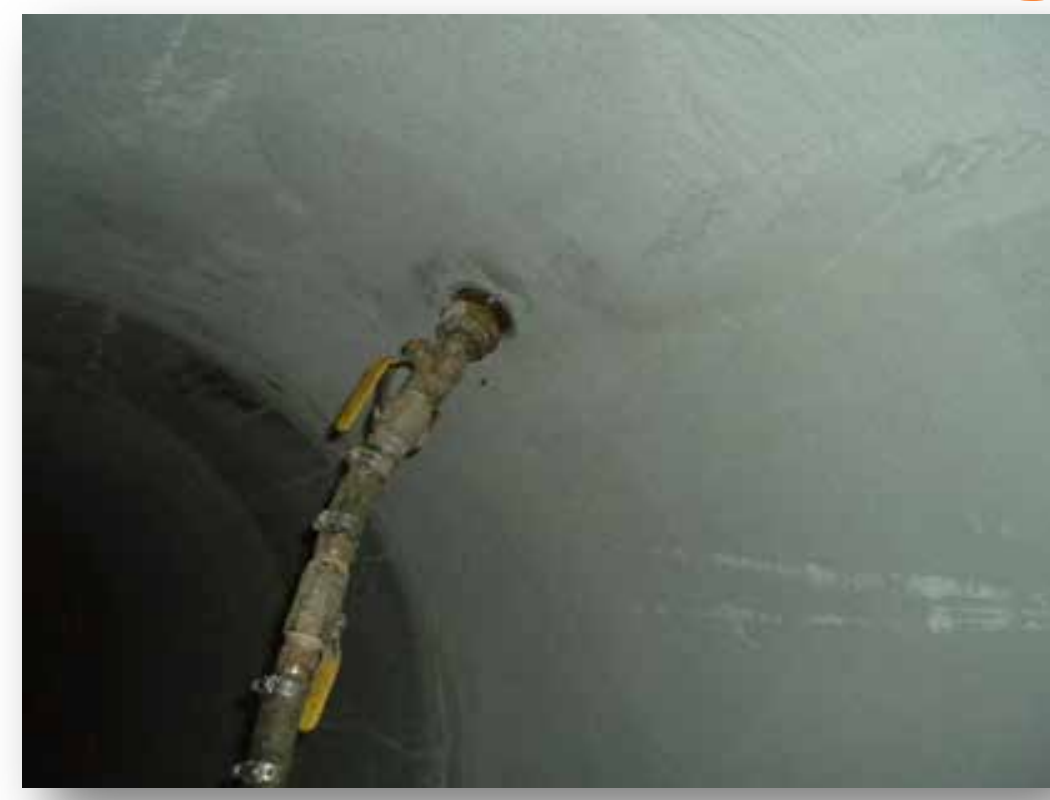

\section{Lubrication}

- Place in overbore annulus to reduce friction (pipe \& soil)

- Water

- Bentonite

- Polymer

- Through grout ports or at front shield

- Up to $50 \%$ reduction to required jacking forces 


\section{Before:}

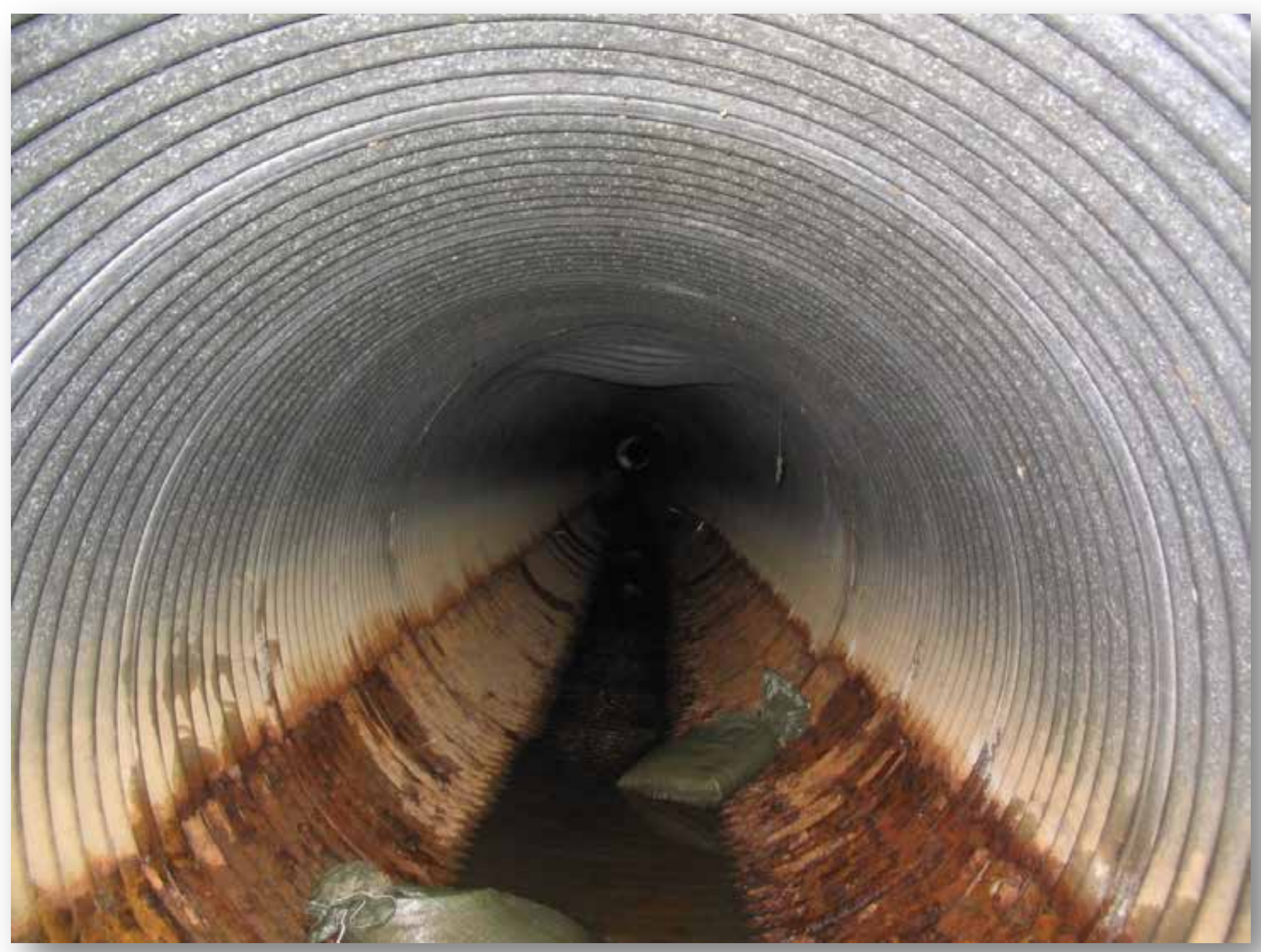

Existing 48" CMP Culvert

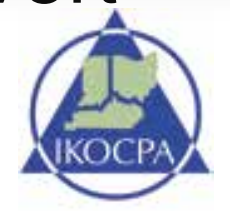




\section{After:}

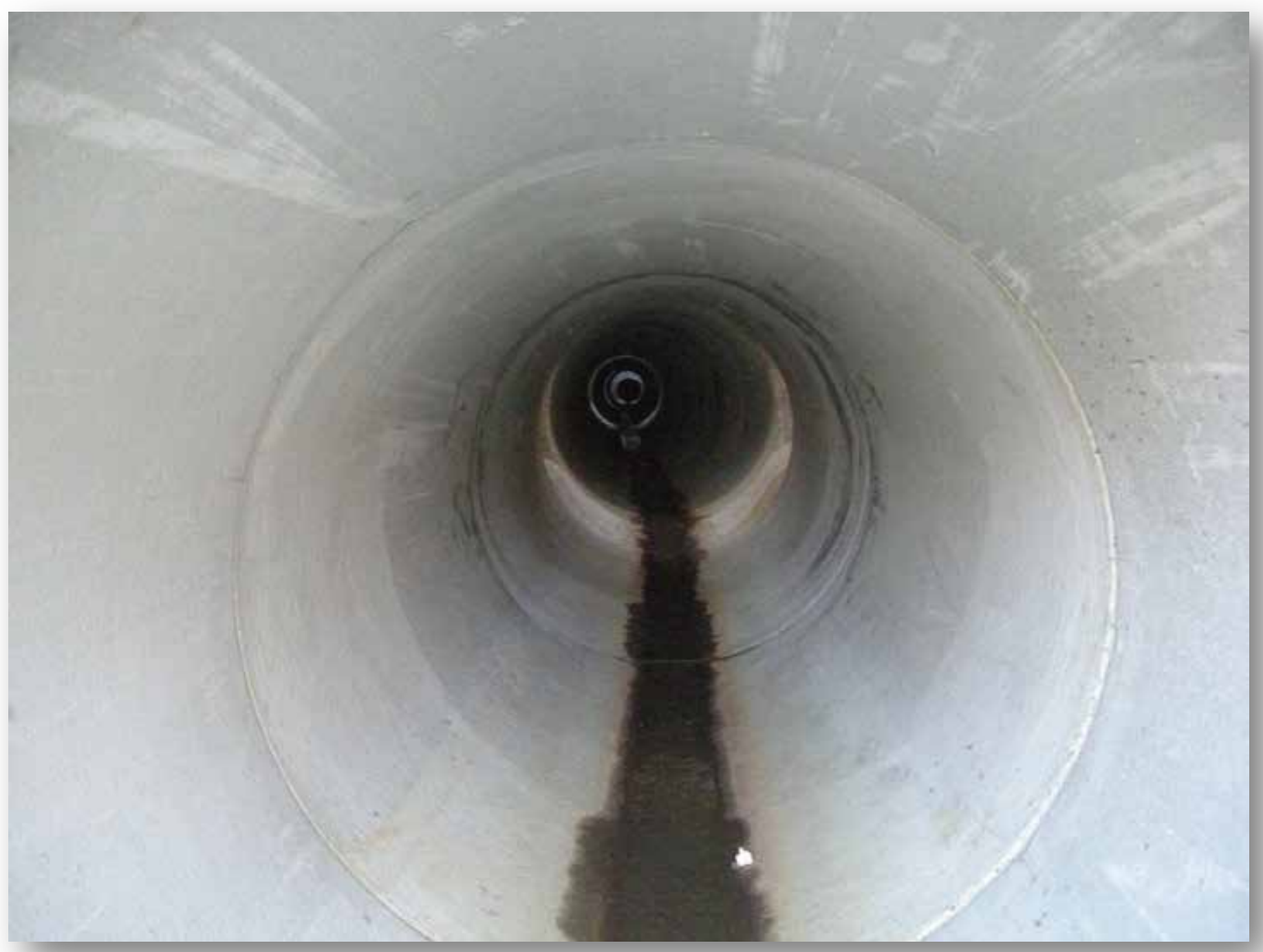

New 54" RCP

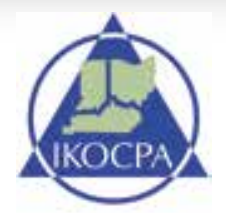




\section{Project Completion}

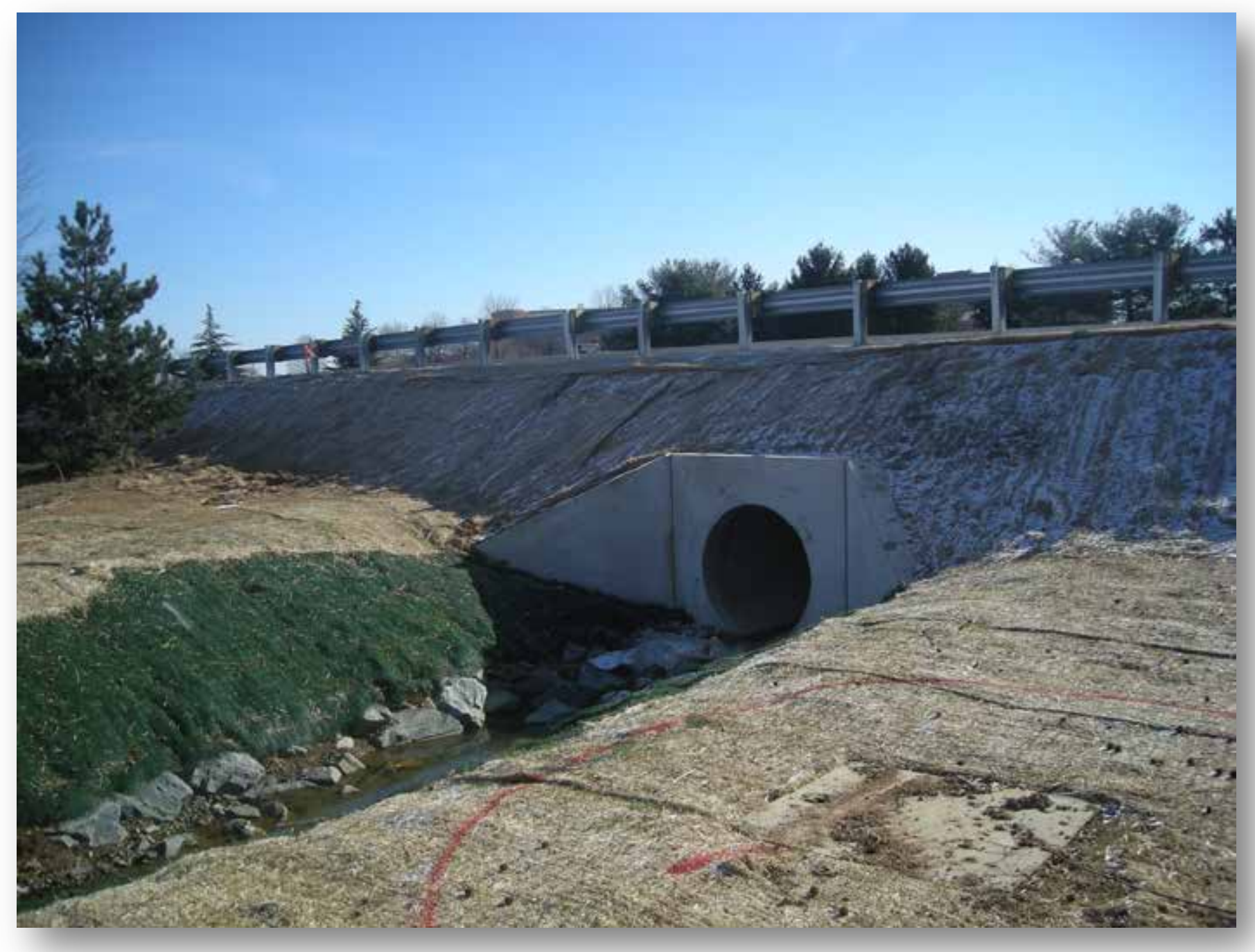

$$
\text { (20) }
$$




\section{Years Later}
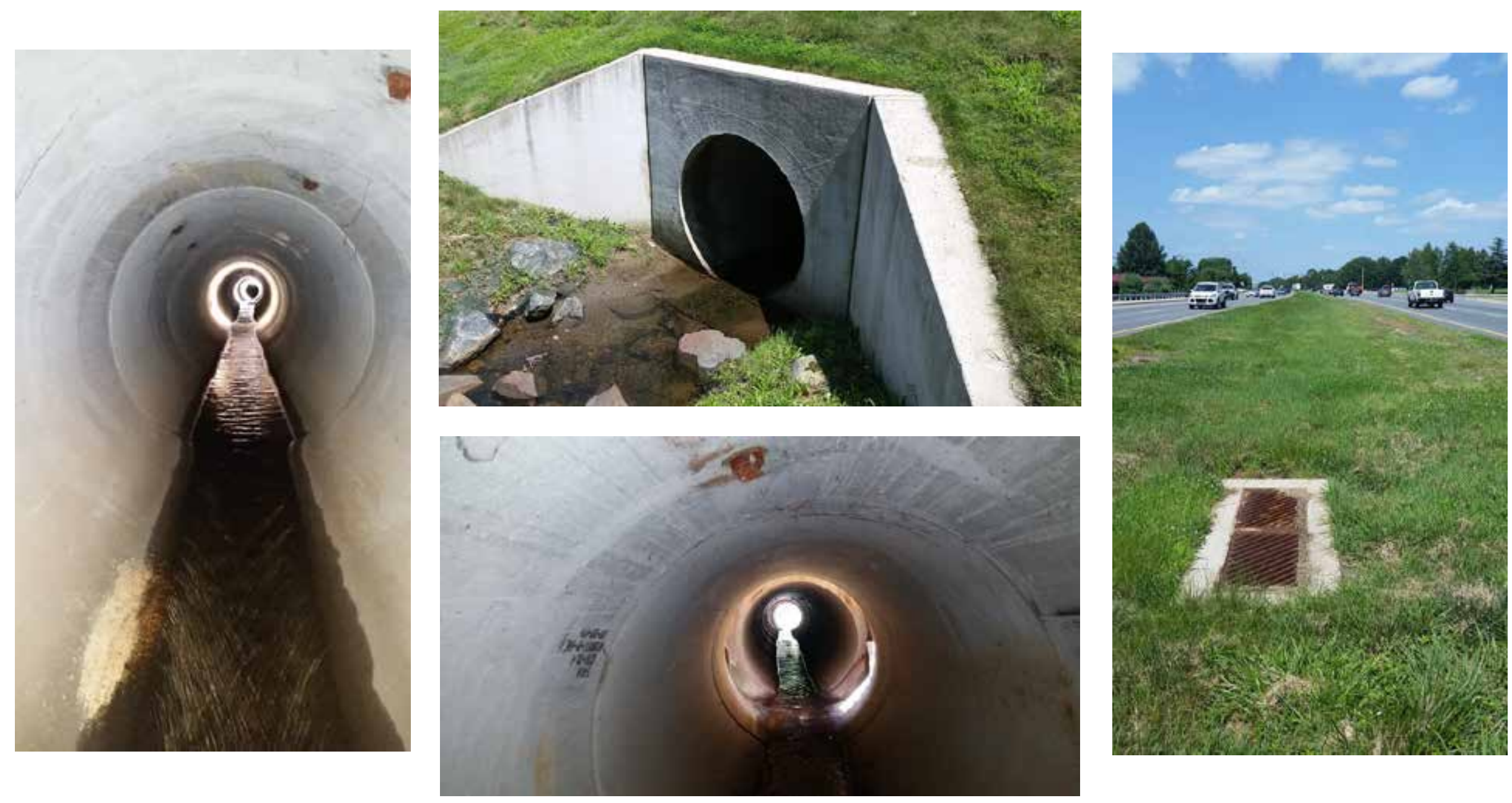

5 


\section{Additional Options}
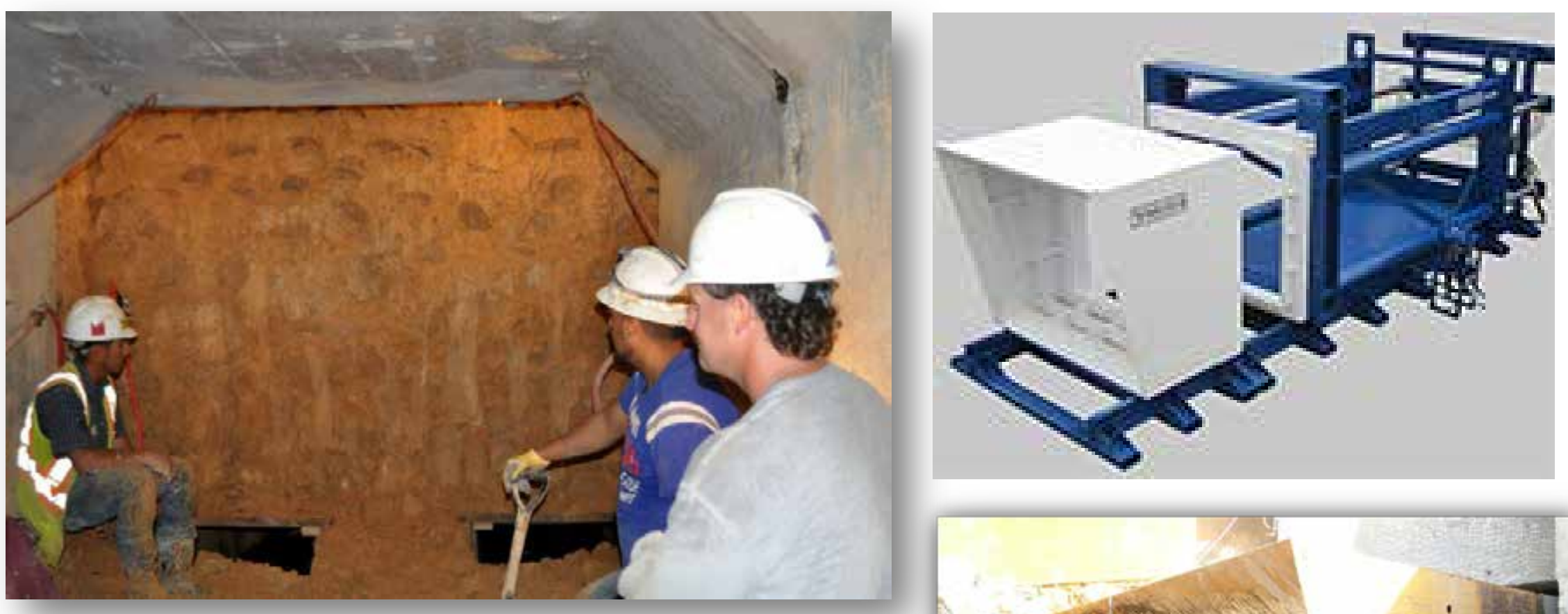

\section{Box Jacking Equipment}

- Box shields \& jacking stations for even more hydraulic capacity

- Shield slightly larger than outside pipe dimensions

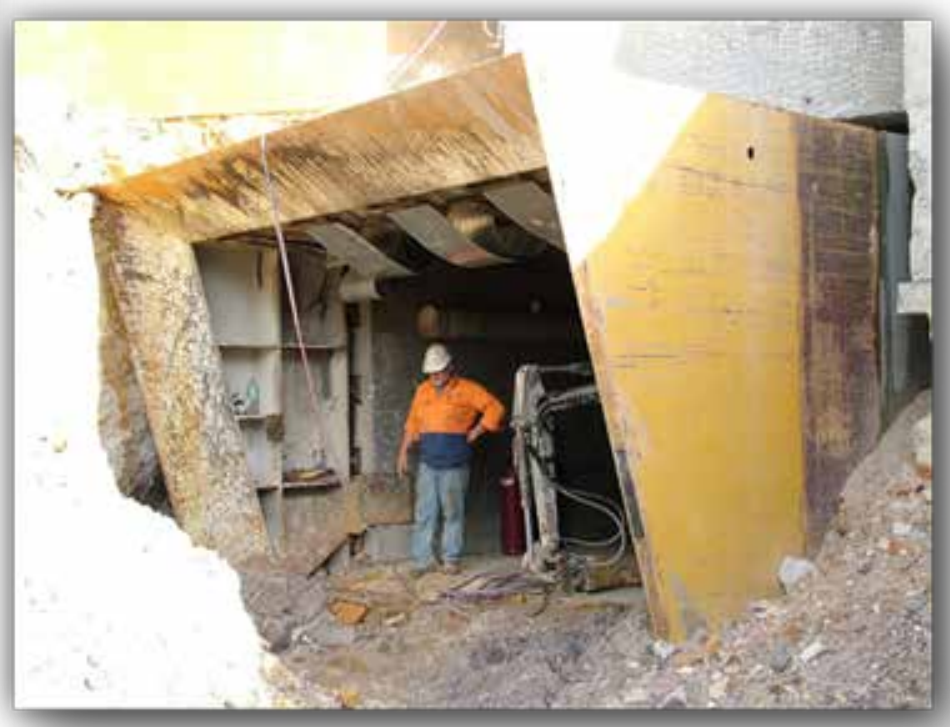


